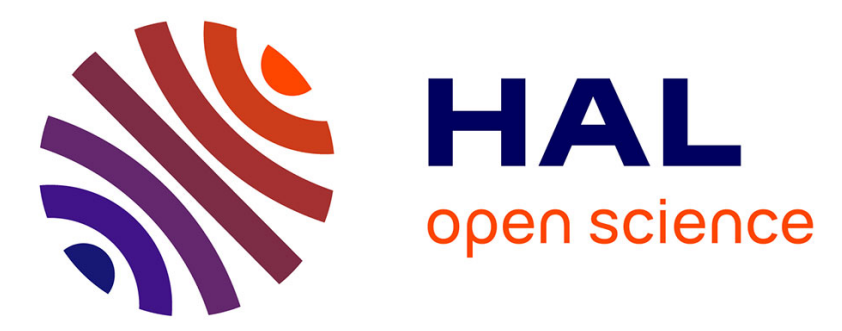

\title{
Reduced-order models for nonlinear vibrations of fluid-filled circular cylindrical shells: Comparison of POD and asymptotic nonlinear normal modes methods
} M. Amabili, Cyril Touzé

\section{To cite this version:}

M. Amabili, Cyril Touzé. Reduced-order models for nonlinear vibrations of fluid-filled circular cylindrical shells: Comparison of POD and asymptotic nonlinear normal modes methods. Journal of Fluids and Structures, 2007, 23 (6), pp.885-903. 10.1016/j.jfluidstructs.2006.12.004 . hal-00838880

\section{HAL Id: hal-00838880}

\section{https://hal-ensta-paris.archives-ouvertes.fr/hal-00838880}

Submitted on 18 Mar 2016

HAL is a multi-disciplinary open access archive for the deposit and dissemination of scientific research documents, whether they are published or not. The documents may come from teaching and research institutions in France or abroad, or from public or private research centers.
L'archive ouverte pluridisciplinaire $\mathbf{H A L}$, est destinée au dépôt et à la diffusion de documents scientifiques de niveau recherche, publiés ou non, émanant des établissements d'enseignement et de recherche français ou étrangers, des laboratoires publics ou privés. 


\title{
Reduced-order models for nonlinear vibrations of fluid-filled circular cylindrical shells: Comparison of POD and asymptotic nonlinear normal modes methods
}

\author{
M. Amabili ${ }^{\mathrm{a}, *}$, C. Touzé ${ }^{\mathrm{b}}$ \\ ${ }^{a}$ Dipartimento di Ingegneria Industriale, Università di Parma, Parco Area delle Scienze 181/A, Parma 43100, Italy \\ ${ }^{\mathrm{b}}$ ENSTA-UME, Unité de Recherche en Mécanique, Chemin de la Hunière, 91761 Palaiseau, Cedex, France
}

Received 5 April 2006; accepted 28 December 2006

Available online 19 March 2007

\begin{abstract}
The aim of the present paper is to compare two different methods available for reducing the complicated dynamics exhibited by large amplitude, geometrically nonlinear vibrations of a thin shell. The two methods are: the proper orthogonal decomposition (POD), and an asymptotic approximation of the nonlinear normal modes (NNMs) of the system. The structure used to perform comparisons is a water-filled, simply supported circular cylindrical shell subjected to harmonic excitation in the spectral neighbourhood of the fundamental natural frequency. A reference solution is obtained by discretizing the partial differential equations (PDEs) of motion with a Galerkin expansion containing 16 eigenmodes. The POD model is built by using responses computed with the Galerkin model; the NNM model is built by using the discretized equations of motion obtained with the Galerkin method, and taking into account also the transformation of damping terms. Both the POD and NNMs allow to reduce significantly the dimension of the original Galerkin model. The computed nonlinear responses are compared in order to verify the accuracy and the limits of these two methods. For vibration amplitudes equal to 1.5 times the shell thickness, the two methods give very close results to the original Galerkin model. By increasing the excitation and vibration amplitude, significant differences are observed and discussed. The response is investigated also for a fixed excitation frequency by using the excitation amplitude as bifurcation parameter for a wide range of variation. Bifurcation diagrams of Poincaré maps obtained from direct time integration and calculation of the maximum Lyapunov exponent have been used to characterize the system.
\end{abstract}

Keywords: Proper orthogonal decomposition; Nonlinear normal modes; Cylindrical shells; Nonlinear vibration; Fluid-filled shells

\section{Introduction}

Reduced-order models (ROMs) are an attractive research topic in nonlinear dynamics of fluid and solid systems. By far, the two most popular methods used to build ROMs are the proper orthogonal decomposition (POD) and 
the nonlinear normal modes (NNMs) methods. The first one (POD, also referred to as the Karhunen-Loève method) uses a cloud of points in phase space, obtained from simulations or from experiments, in order to build the reduced subspace that will contain most information (Zahorian and Rothenberg, 1981; Aubry et al., 1988; Sirovich, 1987; Breuer and Sirovich, 1991; Georgiou et al., 1999; Azeez and Vakakis, 2001; Sarkar and Païdoussis, 2003, 2004; Kerschen et al., 2003, 2005; Amabili et al., 2003, 2006; Georgiou, 2005). The method is, in essence, linear, as it furnishes the best orthogonal basis, which decorrelates the signal components and maximizes variance.

Amabili et al. (2003, 2006) compared Galerkin and POD models of a water-filled circular cylindrical shell from moderate to extremely large vibration amplitudes. Accurate POD models can be built by using only POD modes with significant energy. In particular, Amabili et al. (2006) found that more proper orthogonal modes (POMs) are necessary to reach energy convergence using time series extracted from more complex responses (chaotic or quasi-periodic) than from the periodic ones. Therefore, by using complex responses it is possible to build models with larger dimension, suitable to describe with accuracy large variations of the system parameters.

The second method (NNMs), constructs and defines the researched subspaces from specific properties of the dynamical systems, by adapting the reduction theorems provided by the mathematics: centre manifold theorem (Carr, 1981; Guckenheimer and Holmes, 1983) and normal form theory (Poincaré, 1892; Iooss and Adelmeyer, 1998; Elphick et al., 1987). Their application to vibratory systems led to two definitions of NNMs, which are equivalent in a conservative framework: either a family of periodic orbits in the vicinity of the equilibrium point (Rosenberg, 1966; Mikhlin, 1995; Vakakis et al., 1996), or an invariant manifold containing these periodic orbits (Shaw and Pierre, 1991). Numerous asymptotic methods have been proposed for their computation, by application of the centre manifold theorem (Shaw and Pierre, 1993), the normal form theory (Jézéquel and Lamarque, 1991; Touzé et al., 2004; Touzé and Amabili, 2006), the conservation of energy for conservative systems (King and Vakakis, 1994), or the method of multiple scales (Lacarbonara et al., 2003). Numerical procedures have also been proposed, recently by Jiang et al. (2005a, b), who extended the method proposed by Pesheck et al. (2002) for conservative cases. Bellizzi and Bouc (2005) propose a numerical resolution of an extended KBM (Krylov-Bogoliubov-Mitropolsky) method, while Slater (1996) used continuation techniques to generate the NNM.

Application of the POD and NNMs methods to reduced-order modelling enabled to show that a few degrees of freedom (dof) are generally enough to catch the nonlinear behaviour of many structures, versus the several necessary in the corresponding Galerkin models.

The aim of the present study is to provide a full comparison of the results given by the two reduction methods on a realistic example: a water-filled circular cylindrical shell. The reference solution is obtained by the Galerkin method. Its convergence has been carefully checked (Pellicano et al., 2002), and comparisons with experiments have been performed (Amabili, 2003). The construction of the POD model has been exhaustively explained in Amabili et al. (2003, 2006), whereas the asymptotic NNMs procedure used here is fully explained in Touze and Amabili (2006). The peculiarity of the NNMs formulation is that damping is taken into account via an improvement of the real normal form calculation presented in Touzé et al. (2004). Comparisons will be drawn on two different cases. First, the ability of the methods to recover frequency-response curves will be investigated, for moderate values of the amplitude of the external force. Then, bifurcation diagrams for varying amplitude of the forcing, leading to complex dynamics, will be discussed.

\section{Basic equations for nonlinear vibrations of shells}

A cylindrical coordinate system $(\mathrm{O} ; x, r, \theta)$ is chosen, with the origin $\mathrm{O}$ placed at the centre of one end of the shell. The displacements of the middle surface of the shell are denoted by $u, v$ and $w$, in the axial, circumferential and radial directions, respectively; $w$ is taken positive inwards. By using Donnell's nonlinear shallow-shell theory, the equation of motion for finite-amplitude transverse deflection is given by (Evensen, 1967; Amabili and Païdoussis, 2003)

$$
D \nabla^{4} w+c h \dot{w}+\rho h \ddot{w}=f-p+\frac{1}{R} \frac{\partial^{2} F}{\partial x^{2}}+\frac{1}{R^{2}}\left[\frac{\partial^{2} F}{\partial \theta^{2}}\left(\frac{\partial^{2} w}{\partial x^{2}}\right)-2 \frac{\partial^{2} F}{\partial x \partial \theta}\left(\frac{\partial^{2} w}{\partial x \partial \theta}\right)+\frac{\partial^{2} F}{\partial x^{2}}\left(\frac{\partial^{2} w}{\partial \theta^{2}}\right)\right],
$$

where $D=E h^{3} /\left[12\left(1-v^{2}\right)\right]$ is the flexural rigidity; $E$, the Young's modulus; $v$, the Poisson ratio; $h$, the shell thickness; $R$, the mean shell radius; $\rho$, the mass density of the shell; $c$, the coefficient of viscous damping; $p$, the radial pressure applied to the surface of the shell by the contained fluid, and $f$ is an external local excitation:

$$
f=\tilde{f} \delta(R \theta-R \tilde{\theta}) \delta(x-\tilde{x}) \cos (\omega t),
$$


where $\delta$ is the Dirac delta function, $\tilde{f}$ is the magnitude of the localized (point) force, and $\tilde{\theta}$ and $\tilde{x}$ give the angular and axial coordinates of the point of application of the force, respectively. The viscous damping model introduced in Eq. (1) is replaced by modal damping coefficients in the equations of motion.

In Eq. (1) the overdot denotes a time derivative, and $F$ is the in-plane Airy stress function, which is given by the following compatibility equation (Evensen, 1967; Amabili and Païdoussis, 2003):

$$
\frac{1}{E h} \nabla^{4} F=-\frac{1}{R} \frac{\partial^{2} w}{\partial x^{2}}+\frac{1}{R^{2}}\left[\left(\frac{\partial^{2} w}{\partial x \partial \theta}\right)^{2}-\frac{\partial^{2} w}{\partial x^{2}} \frac{\partial^{2} w}{\partial \theta^{2}}\right] .
$$

In Eqs. (1) and (3), the biharmonic operator is defined as $\nabla^{4}=\left[\partial^{2} / \partial x^{2}+\partial^{2} /\left(R^{2} \partial \theta^{2}\right)\right]^{2}$. Donnell's nonlinear shallowshell equations are accurate only for modes with $n \geqslant 4$.

Attention is focused on simply supported, circumferentially closed circular cylindrical shells of length $L$. The following out-of-plane (shell surface) boundary conditions are imposed:

$$
w=0, \quad M_{x}=-D\left\{\left(\partial^{2} w / \partial x^{2}\right)+v\left[\partial^{2} w /\left(R^{2} \partial \theta^{2}\right)\right]\right\}=0 \text { at } x=0, L,
$$

where $M_{x}$ is the bending moment per unit length. The in-plane boundary conditions are

$$
N_{x}=0 \quad \text { and } \quad v=0 \text { at } x=0, L,
$$

where $N_{x}$ is the force per unit length in axial direction. In addition, $u, v$ and $w$ must be continuous in $\theta$.

Excitations with frequency close to the natural frequency of the lowest modes of the shell are considered; low-frequency modes are associated with predominantly radial motion and are identified by the pair $(m, n)$, where $m$ is the number of axial half-waves and $n$ is the number of circumferential waves.

\subsection{Fluid-structure interaction}

The contained fluid is assumed to be incompressible, inviscid and irrotational, so that potential flow theory can be used to describe fluid motion. Liquid-filled shells vibrating in the low-frequency range satisfy the incompressibility hypothesis very well. Nonlinear effects in the dynamic pressure and in the boundary conditions at the fluid-structure interface are neglected, which is a very good approximation for boundary displacements of two orders of magnitude (or more) smaller than the diameter of the fluid domain. The shell prestress due to the fluid weight is also neglected. The fluid motion is described by the velocity potential $\Phi$, which satisfies the Laplace equation; cavitation is assumed not to occur. Both ends of the fluid volume, corresponding to the shell edges, are assumed to be open, so that a zero pressure is imposed there; this physically corresponds to a long shell periodically supported (e.g. with ring stiffeners) or it approximates a shell closed by very thin circular plates. The dynamic pressure $p$ exerted by the contained fluid on the shell is given by Amabili (2003):

$$
p=\rho_{F}(\dot{\Phi})_{r=R}=\sum_{m=1}^{M} \sum_{n=1}^{N} \rho_{F}\left[\ddot{A}_{m, n}(t) \cos (n \theta)+\ddot{B}_{m, n}(t) \sin (n \theta)\right] \frac{I_{n}\left(\lambda_{m} R\right)}{\lambda_{m} I_{n}^{\prime}\left(\lambda_{m} R\right)} \sin \left(\lambda_{m} x\right),
$$

where $\rho_{F}$ is the mass density of the internal fluid, $A_{m n}(t)$ and $B_{m n}(t)$ are the generalized coordinates describing the shell oscillation (see Eq. (8) for more details), overdots indicate time derivatives, $I_{n}$ is the modified Bessel function of order $n$, and $I_{n}^{\prime}$ its derivative with respect to the argument.

\section{Reference solution and reduced-order models}

\subsection{Galerkin method}

The Galerkin method, employing any set of basis functions $\varphi_{i}$, approximates the nonlinear partial differential equation (PDE) by transforming it into a finite set of coupled ordinary differential equations (ODEs), with the solution being expressed as

$$
w(\xi, t)=\sum_{i=1}^{K} q_{i}(t) \varphi_{i}(\xi)
$$

where $t$ is time, $\xi$ is the vector of spatial coordinate $(x, \theta)$ describing the shell middle surface $\Omega, q_{i}(t)$ are the generalized coordinates, and $K$ is the number of generalized coordinates (dof), i.e. the number of basis functions 
assumed. The linear modal base is the best choice for discretizing the shell, as these are the eigenfunctions of the linear operator of the PDE. The orthogonality property of the eigenmodes allows decoupling the ODEs at the linear stage. Other sets of basis functions may be used, with the consequence that the ODEs are linearly coupled, and more functions are needed to attain convergence. The key question in the Galerkin method is the convergence of the solution. In order to have a reasonable number of dof, it is important to use the most significant modes. In addition to the asymmetric mode directly driven into vibration by the excitation (driven mode), it is necessary to consider (i) the orthogonal mode having the same shape and natural frequency but rotated by $\pi /(2 n)$ (companion mode), (ii) additional asymmetric modes, and (iii) axisymmetric modes. In fact, it has clearly been established that, for large-amplitude shell vibrations, the deformation of the shell involves significant axisymmetric oscillations inwards. According to these considerations, the radial displacement $w$ is expanded by using the eigenmodes of the empty shell, which are unchanged for the completely filled shell (Amabili, 2003):

$$
w(x, \theta, t)=\sum_{m=1}^{3} \sum_{k=1}^{3}\left[A_{m, k n}(t) \cos (k n \theta)+B_{m, k n}(t) \sin (k n \theta)\right] \sin \left(\lambda_{m} x\right)+\sum_{m=1}^{4} A_{(2 m-1), 0}(t) \sin \left(\lambda_{(2 m-1)} x\right),
$$

where $k n$ is the number of circumferential waves, $m$ is the number of longitudinal half-waves (only odd values are used for symmetry), $\lambda_{m}=m \pi / L$, and $t$ is the time; $A_{m, n}(t), B_{m, n}(t)$ and $A_{m, 0}(t)$ are the generalized coordinates that are unknown functions of $t$; the mode driven in resonance is $(1, n)$, i.e. the mode for $m=k=1$. The number of dof used in the present numerical calculations is 16 (Amabili, 2003).

The presence of pairs of modes having the same shape but different angular orientations, the first one described by $\cos (n \theta)$ (driven mode for the excitation given by Eq. (2)) and the other by $\sin (n \theta)$ (companion mode), in the periodic response of the shell leads to the appearance of a travelling wave around the shell in the $\theta$ direction when both these modes are active and when they have a relative time shift. This phenomenon is due to the axial symmetry of the system.

When the excitation has a frequency close to the resonance of a particular mode, say $(m=1, n)$, results for relatively low amplitude excitation (case of periodic response) show that (i) the generalized coordinates $A_{1, n}(t)$ and $B_{1, n}(t)$ have the same frequency as the excitation, (ii) the coordinates $A_{1,2 n}(t), B_{1,2 n}(t), A_{3,2 n}(t), B_{3,2 n}(t)$ and all the coordinates associated with axisymmetric modes have twice the frequency of the excitation, and (iii) the coordinates $A_{3, n}(t), B_{3, n}(t), A_{1,3 n}(t)$, $B_{1,3 n}(t), A_{3,3 n}(t)$ and $B_{3,3 n}(t)$ have three times the frequency of the excitation.

Expansion (8) used for the radial displacement $w$ satisfies identically the boundary conditions given by Eqs. (4a,b); moreover, it satisfies exactly the continuity of the circumferential displacement. The boundary conditions for the inplane displacements, Eqs. (5a,b), give very complex expressions when transformed into equations involving $w$. Therefore, they are modified into simpler integral expressions that satisfy Eqs. (5a,b) on the average (Amabili, 2003).

When the expansion of $w$, Eq. (8), is substituted in the right-hand side of Eq. (3), a PDE for the stress function $F$ is obtained, composed of the homogeneous and the particular solution.

Eqs. (6) and (8) present the same spatial distribution on the shell surface. Therefore, the fluid pressure gives only an inertial effect, which is different for each mode of the expansion. Hence, the fluid is expected to change the nonlinear behaviour of the fluid-filled shell, as a consequence of the fundamental interaction among asymmetric and the axisymmetric modes. Usually the inertial effect of the fluid is larger for axisymmetric modes, thus enhancing the nonlinear softening-type behaviour of the shell.

By use of the Galerkin method, 16 second-order, ordinary, coupled nonlinear differential equations are obtained for the variables $A_{m, k n}(t), B_{m, k n}(t)$ and $A_{m, 0}(t)$, for $m=1, \ldots, M$ and $k=1, \ldots, 3$, by successively weighting the original equation (1) with the functions that describe the shape of the modes retained in Eq. (8). These equations have very long expressions containing quadratic and cubic nonlinear terms and have been obtained by using the Mathematica 4 computer software (Wolfram, 1999), in order to perform analytical integrals of trigonometric functions. The generic $j$ th Lagrange equation is divided by the modal mass associated with $\ddot{q}_{j}$, taking the following form:

$$
\ddot{q}_{j}+\omega_{j}^{2} q_{j}+2 \zeta_{j} \omega_{j} \dot{q}_{j}+\sum_{i=1}^{K} \sum_{p=1}^{K} g_{i p}^{(j)} q_{i} q_{p}+\sum_{i=1}^{K} \sum_{p=1}^{K} \sum_{k=1}^{K} h_{i p k}^{(j)} q_{i} q_{p} q_{k}=\hat{f}_{j} \cos (\omega t) \quad \text { for } j=1, \ldots, K,
$$

where $\hat{f}_{j}$ is projection of the nondimensionalized force, which must be set equal to zero in all the equations where $q_{j}=B_{m, k n}, g_{i p}^{(j)}$ are coefficients of quadratic terms and $h_{i p k}^{(j)}$ are coefficients of cubic terms; $\zeta_{j}$ is the modal damping ratio, replacing here the unrealistic viscous damping introduced in Eq. (1). In Eq. (9) each generalized coordinate $q_{j}$ (and therefore the modal damping $\zeta_{j}$ and circular frequency $\left.\omega_{j}\right)$ has to be referred to mode $(m, n)$, i.e. $q_{j}=A_{m, n}$ or $B_{m, n}$. For computational convenience a nondimensionalization of variables is also performed: the time is divided by the period of the resonant mode and the vibration amplitudes are divided by the shell thickness $h$. It can be observed that nonlinear terms do not involve time derivatives of $q_{j}$. By introducing a dummy variable, the $K$ second-order equations are 
transformed into $2 \times K$ first-order nonlinear differential equations that are studied by using (i) the software AUTO 97 (Doedel et al., 1998) for continuation and bifurcation analysis of nonlinear ODEs, and (ii) direct integration of the equations of motion by using the DIVPAG routine of the Fortran library IMSL. Continuation methods allow following the solution path, with the advantage that unstable solutions can also be obtained; these are not ordinarily attainable by using direct numerical integration. The software AUTO 97 is capable of continuation of the solution, bifurcation analysis and branch switching by using pseudo-arclength continuation and collocation methods.

Direct integration of the equations of motion by using Gear's BDF method (routine DIVPAG of the Fortran library IMSL) has also been performed, when specified, to check the results and obtain the time behaviour. Gear's algorithm was used due to the relatively high dimension of the dynamical system.

The bifurcation diagram of the Poincare maps was used in case of nonstationary response, i.e. to analyse a wide range of excitation magnitudes where the shell response changes dramatically. This bifurcation diagram has been constructed by using the time integration scheme and by varying the force amplitude.

\subsection{Proper orthogonal decomposition (POD) method}

The POD method optimally extracts the spatial information necessary to characterize the spatio-temporal complexity and inherent dimension of a system, from a set of temporal snapshots of the response, gathered from either numerical simulations or experimental data. In the present context, the temporal responses are obtained via the conventional Galerkin solution. The POMs obtained by the POD method will be used as a basis in conjunction with the Galerkin approach. The solution can be expressed by using the basis of the POMs $\psi_{i}(\xi)$,

$$
w(\xi, t)=\sum_{i=1}^{\tilde{K}} a_{i}(t) \psi_{i}(\xi)
$$

where $a_{i}$ are the proper orthogonal coordinates and $\tilde{K}$ is the number of POMs (dof) used to build the POD model (in general, significantly lower than $K$ in Eq. (7) necessary for the conventional Galerkin method).

The displacement field $w$ is divided into its time-mean value $\bar{w}(\xi)$ and the zero-mean response $\tilde{w}(\xi, t)=$ $(w(\xi, t)-\bar{w}(\xi))$. In the POD method, the POMs are obtained by minimizing the objective function

$$
\tilde{\lambda}=\left\langle(\psi(\xi)-\tilde{w}(\xi, t))^{2}\right\rangle \quad \forall \xi \in \Omega,
$$

with \langle\rangle denoting the time-averaging operation and $\psi(\xi)$ the generic POD mode. If the temporal snapshots of $\tilde{w}$ are denoted by $\left\{\tilde{w}_{n}\right\}$, the time-averaging operation of a series of $N$ snapshots is $\langle\tilde{w}(\xi, t)\rangle=(1 / N) \sum_{n=1}^{N} \tilde{w}_{n}(\xi)$. Minimizing of the objective function (11) is obtained, after some mathematics, by solving the following eigenvalue problem:

$$
\int_{\Omega}\left\langle\tilde{w}(\xi, t) \tilde{w}\left(\xi^{\prime}, t\right)\right\rangle \psi\left(\xi^{\prime}\right) \mathrm{d} \xi^{\prime}=\lambda \psi(\xi),
$$

where $\left\langle\tilde{w}(\xi, t) \tilde{w}\left(\xi^{\prime}, t\right)\right\rangle$ is the time-averaged spatial autocorrelation function.

A Galerkin projection scheme for determining POMs semi-analytically (Sarkar and Païdoussis, 2003), and in parallel to approximate the solution of the PDE, is presented next. The generic POM is projected on the eigenmodes $\varphi_{i}(\xi)$ of the shell as

$$
\psi(\xi)=\sum_{i=1}^{K} \alpha_{i} \varphi_{i}(\xi)
$$

where $\alpha_{i}$ are unknown coefficients. Then, the following eigenvalue problem is finally obtained:

$$
\mathbf{A} \boldsymbol{\alpha}=\lambda \mathbf{B} \boldsymbol{\alpha},
$$

where

$$
A_{i j}=\tau_{i} \tau_{j}\left\langle\tilde{q}_{i}(t) \tilde{q}_{j}(t)\right\rangle, \quad B_{i j}=\tau_{i} \delta_{i j}, \quad \tau_{i}=\int_{\Omega} \varphi_{i}^{2}(\xi) \mathrm{d} \xi,
$$

$\delta_{i j}$ is the Kronecker delta, $\tilde{q}_{i}=\left(q_{i}-\bar{q}_{i}\right)$ is the zero-mean response of the $i$ th generalized coordinate, with $\bar{q}_{i}$ being its mean. The norm of the basis functions $\tau_{i}$ in the present case is $\pi R L / 2$ for asymmetric modes and $\pi R L$ for axisymmetric modes; without effect on the results, they can be assumed to be 0.5 and 1, respectively. In Eq. (14), $\mathbf{A}$ and $\mathbf{B}$ are symmetric and positive definite matrices of dimension $K \times K$, and $\alpha$ is a vector containing the $K$ unknown coefficients of the POMs. The eigenvectors $\alpha$ corresponding to the largest eigenvalues (known as dominant POMs) in Eq. (14) can now be inserted in Eq. (13) that gives a basis for the approximate solution of the PDE using the Galerkin approach; this 
will be referred to as the POD-Galerkin scheme hereafter. The optimal number of terms $\tilde{K}$ to be retained can be estimated by $\sum_{i=1}^{\tilde{K}} \lambda_{i} / \sum_{i=1}^{K} \lambda_{i} \geqslant 0.99$ in Eq. (14); in fact, this expression gives an evaluation of the system energy associated with $\tilde{K}$ POMs with respect to the total energy of the system; for each problem this cut-off value can be different. It would be useful to check the convergence of the solution by increasing the value $\tilde{K}$; over a certain value, the results can become less accurate, because the additional terms introduced in the expansion may be highly noisepolluted. In particular, Amabili et al. (2006) found that more POMs are necessary to reach energy convergence using time series extracted from chaotic or quasiperiodic responses than from the periodic ones. Therefore, by using complex responses it is possible to build ROM with larger dimension, suitable for describing with accuracy large variations of the system parameters, even if they give a less-effective reduction of the order of the system.

In some applications, it may be better to use time responses obtained for different system parameters in order to produce better proper orthogonal modes.

By using Eqs. (7), (8) and (13), the expansion used for the POD solution is given by

$$
w(\xi, t)=\sum_{i=1}^{\tilde{K}} a_{i}(t) \sum_{j=1}^{K} \alpha_{j, i} \varphi_{j}(\xi)=\sum_{i=1}^{\tilde{K}} a_{i}(t) \sum_{m=1}^{M} \sum_{n=0}^{N}\left[\alpha_{m, n, i} \cos (n \theta)+\beta_{m, n, i} \sin (n \theta)\right] \sin \left(\lambda_{m} x\right),
$$

where on the right-hand side two different symbols, $\alpha_{m, n, i}$ and $\beta_{m, n, i}$, have been introduced to differentiate the coefficients of the POMs for cosine and sine terms in $\theta$ and are given by the corresponding $\alpha_{j, i}$. Eq. (18) is used to solve Eqs. (1) and (3) with the Galerkin method to find the equations of motion of the ROM. Moreover, Eq. (18) has still the same shape over the shell surface as Eq. (6); therefore, the fluid-structure interaction can be treated with the same approach used for the Galerkin method. This is not surprising, because the POD modes have been projected on the eigenmodes.

The POD method gives a set of equations of motion with exactly the same structure of those obtained with the conventional Galerkin method, Eq. (9), with the difference of a reduced order of dof, i.e. $\tilde{K}<K$. Table 1 gives the coefficients $\alpha_{m, n, i}$ and $\beta_{m, n, i}$ for two POD models built from time response obtained from the conventional Galerkin model (i) for $\omega / \omega_{1, n}=0.991$ and $\tilde{f}=3 \mathrm{~N}$ (quasi-periodic response), and (ii) for $\omega / \omega_{1, n}=0.92$ and $\tilde{f}=550 \mathrm{~N}$ (chaotic response); in particular, model (i) has $\tilde{K}=3$ dof and model (ii) has $\tilde{K}=5$ dof, versus the $K=16$ dof of the original Galerkin model. Both travelling waves around the shell in opposite directions have been taken into account (one obtained by direct integration and the other by changing the sign to the generalized coordinates associated to $\sin (n \theta)$ terms in Eq. (8)) to construct the POD ROM; these two time records have been equally weighted in order to construct the matrix A used to extract the eigenvalues in Eq. (14); this is fundamental in reproducing the axisymmetry of the shell with no preferential direction.

\subsection{Asymptotic nonlinear normal modes (NNMs) method}

NNMs are here defined as invariant manifolds of the state space. They are moreover chosen tangent at the origin, which corresponds to the position of the structure at rest. An asymptotic procedure, based on the normal form theory, is used to compute the NNMs of the system. The method is here briefly recalled, the interested reader is referred to Touzé and Amabili (2006) for a complete description. In particular, it allows to take modal damping into account in the derivations, hence extending previous results obtained for conservative systems (Touzé et al., 2004). A third-order asymptotic development is applied, in order to perform a nonlinear change of coordinates for the system of the damped unforced equation of motion, corresponding to Eq. (9) with the right-hand side equal to zero. A real formulation is

Table 1

Coefficients of the main proper orthogonal modes (POMs)

\begin{tabular}{|c|c|c|c|c|c|c|c|}
\hline Time response & $i$ th POM & $\alpha_{1,5, i}$ & $\beta_{1,5, i}$ & $\alpha_{1,10, i}$ & $\beta_{1,10, i}$ & $\alpha_{1,0, i}$ & $\alpha_{3,0, i}$ \\
\hline \multirow[t]{3}{*}{$\omega / \omega_{1, n}=0.991$} & 1 & 1 & 0 & 0.000213 & 0 & 0.0000434 & $8.85 \times 10^{-6}$ \\
\hline & 2 & 0 & 1 & 0 & -0.000291 & 0 & 0 \\
\hline & 3 & 0.000123 & 0 & -0.1847 & 0 & -0.9641 & 0.1855 \\
\hline \multirow{5}{*}{$\begin{array}{l}\text { Chaotic response, } 550 \mathrm{~N} \text {, } \\
\omega / \omega_{1, n}=0.92\end{array}$} & 1 & 1 & 0 & 0.0006039 & 0 & -0.009812 & 0.002283 \\
\hline & 2 & 0 & 1 & 0 & -0.000273 & 0 & 0 \\
\hline & 3 & -0.02058 & 0 & 0.009702 & 0 & -0.9914 & 0.1143 \\
\hline & 4 & 0 & -0.000873 & 0 & 0.9975 & 0 & 0 \\
\hline & 5 & -0.001390 & 0 & -0.9965 & 0 & -0.003704 & 0.009041 \\
\hline
\end{tabular}


used, so that normal forms are expressed with oscillators. The dummy variable $y_{j}=\dot{q}_{j}$ for the nondimensional velocity permits to recast the system of equations into first order. The nonlinear change of coordinates is

$$
\begin{aligned}
q_{j}= & r_{j}+\sum_{i=1}^{K} \sum_{p \geqslant i}^{K}\left(a_{i p}^{(j)} r_{i} r_{p}+b_{i p}^{(j)} s_{i} s_{p}\right)+\sum_{i=1}^{K} \sum_{p=1}^{K} c_{i p}^{(j)} r_{i} s_{p}+\sum_{i=1}^{K} \sum_{p \geqslant i}^{K} \sum_{k \geqslant p}^{K}\left(d_{i p k}^{(j)} r_{i} r_{p} r_{k}+e_{i p k}^{(j)} s_{i} s_{p} s_{k}\right) \\
& +\sum_{i=1}^{K} \sum_{p=1}^{K} \sum_{k \geqslant p}^{K}\left(t_{i p k}^{(j)} s_{i} r_{p} r_{k}+u_{i p k}^{(j)} r_{i} s_{p} s_{k}\right), \\
y_{j}= & s_{j}+\sum_{i=1}^{K} \sum_{p \geqslant i}^{K}\left(\alpha_{i p}^{(j)} r_{i} r_{p}+\beta_{i p}^{(j)} s_{i} s_{p}\right)+\sum_{i=1}^{K} \sum_{p=1}^{K} \gamma_{i p}^{(j)} r_{i} s_{p}+\sum_{i=1}^{K} \sum_{p \geqslant i}^{K} \sum_{k \geqslant p}^{K}\left(\lambda_{i p k}^{(j)} r_{i} r_{p} r_{k}+\mu_{i p k}^{(j)} s_{i} s_{p} s_{k}\right) \\
& +\sum_{i=1}^{K} \sum_{p=1}^{K} \sum_{k \geqslant p}^{K}\left(v_{i p k}^{(j)} s_{i} r_{p} r_{k}+\xi_{i p k}^{(j)} r_{i} s_{p} s_{k}\right),
\end{aligned}
$$

where $r_{j}$ is the transformed nondimensional displacement and $s_{j}$ is the transformed nondimensional velocity; other symbols are the transformation coefficients. After substitution of (19) into (9), the dynamics, written with the newly introduced variables $\left(r_{j}, s_{j}\right)$, is expressed in an invariant-based span of the state space. As a result, proper truncations can now be realized, as all invariant-breaking terms between oscillators in Eq. (9), have been cancelled. The reduction can now be applied by simply selecting the most important normal coordinates for simulation (master coordinates), and cancelling all the others. In the case considered here, the minimum model must retain the NNMs corresponding to the driven mode $\left(r_{1}, s_{1}\right.$, that are the continuations of $A_{1,5}$ and $\left.\dot{A}_{1,5}\right)$ and the companion mode $\left(r_{2}, s_{2}\right.$, that are the continuations of $B_{1,5}$ and $\dot{B}_{1,5}$ ), as these two modes have the same eigenfrequency (1:1 internal resonance). Finally, the ROM built by selecting these two pairs of coordinates takes the form

$$
\begin{aligned}
\ddot{r}_{1} & +\omega_{1}^{2} r_{1}+2 \zeta_{1} \omega_{1} \dot{r}_{1}+\left(A_{111}^{(1)}+h_{111}^{(1)}\right) r_{1}^{3}+B_{111}^{(1)} r_{1} \dot{r}_{1}^{2}+\left(A_{212}^{(1)}+A_{122}^{(1)}+h_{122}^{(1)}\right) r_{1} r_{2}^{2}+B_{122}^{(1)} r_{1} \dot{r}_{2}^{2} \\
& +B_{212}^{(1)} r_{2} \dot{r}_{1} \dot{r}_{2}+C_{111}^{(1)} r_{1} \dot{r}_{1}+\left(C_{122}^{(1)}+C_{212}^{(1)}\right) r_{1} r_{2} \dot{r}_{2}+C_{221}^{(1)} r_{2}^{2} \dot{r}_{1}=\hat{f} \cos (\omega t), \\
\ddot{r}_{2} & +\omega_{2}^{2} r_{2}+2 \zeta_{2} \omega_{2} \dot{r}_{2}+\left(A_{222}^{(2)}+h_{222}^{(2)}\right) r_{2}^{3}+B_{222}^{2} r_{2} \dot{r}_{2}^{2}+\left(A_{112}^{(2)}+A_{211}^{(2)}+h_{112}^{(2)}\right) r_{2} r_{1}^{2}+B_{211}^{(2)} r_{2} \dot{r}_{1}^{2} \\
& +B_{112}^{(2)} r_{1} \dot{r}_{1} \dot{r}_{2}+C_{222}^{(2)} r_{2}^{2} \dot{r}_{2}+\left(C_{121}^{(2)}+C_{211}^{(2)}\right) r_{1} r_{2} \dot{r}_{1}+C_{112}^{(2)} r_{1}^{2} \dot{r}_{2}=0,
\end{aligned}
$$

where $h_{i p k}^{(j)}$ are the coefficients of cubic terms in Eq. (9), $A_{i p k}^{(j)}, B_{i p k}^{(j)}$ and $C_{i p k}^{(j)}$ arise from the cancellation of the quadratic terms, and are expressed by

$$
A_{i p k}^{(j)}=\sum_{l \geqslant i}^{K} g_{i l}^{(j)} a_{p k}^{(l)}+\sum_{l=1}^{l \leqslant i} g_{l i}^{(j)} a_{p k}^{(l)}, \quad B_{i p k}^{(j)}=\sum_{l \geqslant i}^{K} g_{i l}^{(j)} b_{p k}^{(l)}+\sum_{l=1}^{l \leqslant i} g_{l i}^{(j)} b_{p k}^{(l)}, \quad C_{i p k}^{(j)}=\sum_{l \geqslant i}^{K} g_{i l}^{(j)} c_{p k}^{(l)}+\sum_{l=1}^{l \leqslant i} g_{l i}^{(j)} c_{p k}^{(l)},
$$

where $g_{i l}^{(j)}$ are the coefficients of quadratic terms in Eq. (9). A forcing term has been added at the end of the process and now appears in Eq. (20a). This is the second approximation used for building this ROM, as a time-invariant manifold is used. The most accurate solution would have consisted in constructing a periodically forced invariant manifold, see e.g. Jiang et al. (2005b). However, this results in a very complicated formulation and time-consuming numerical calculations for constructing the ROM. The proposed method has the advantage of simplicity, quickness of computation, and allows deriving a differential model that could be used easily for parametric studies. However, it is valid, strictly speaking, only for small values of $\hat{f}$.

With the NNMs method, the original 16 dof of the conventional Galerkin model have been reduced to two in Eq. (20). However, differently to the POD method, the structure of the equations of motion is changed. In fact, quadratic nonlinear terms have been cancelled, but cubic terms involve both the transformed nondimensional displacement and the transformed nondimensional velocity.

\subsection{Discussion}

After presentation of the two reduction methods, a first discussion on their theoretical settings is here provided in order to underline their abilities and limitations.

The POD method, which consists in finding the best orthogonal hyper-planes that contain most information, is essentially a linear method. This can be seen as an advantage since few manipulations, involving linear algebra only, are needed to construct the ROM. The key formula of the method, Eq. (14), is an eigenvalue problem. On the other hand, 
the linear essence of the method may be a drawback, as curved subspaces are generally more suitable to capture clouds of points with complicated shapes. A NNM, being an invariant manifold in state space, is a curved subspace, so that the NNM reduction method is essentially nonlinear. The invariance property is the key that allows finding the lowest dimensional subspaces that contains dynamical properties, since dynamical motions do not stay within any other subspace that does not share this invariance property. This is the main advantage of the NNMs method as compared to the POD. It is expected that fewer NNMs are necessary than POD modes. This will be illustrated in Sections 4.1 and 4.2 .

The POD method is global in the sense that it is able to capture any motion in state space and furnishes the adapted basis for decomposing it. This is an advantage as compared to the asymptotic NNMs method used here, which relies on a local theory. The third-order development, Eq. (19), is valid for small values of the modal amplitudes. The use of a time-invariant manifold in NNMs can give unreliable results for large values of the amplitude of the external forcing; in fact, the oscillations of the manifold will be too large, and the time-independent transformation will become too crude an approximation. When increasing the nonlinearities by feeding more energy into the system, the results provided by the asymptotic NNMs method are expected to deteriorate. This is not the case for the POD, if one has taken care to construct its POD-based ROM with clouds of points that are significant for a large range of values of the nonlinearity. In this context, it has already been argued (Kerschen et al., 2003; Amabili et al., 2006) that a chaotic response is the best candidate for building the POD.

Finally, the two methods differ radically in the way the ROM is built. For the POD, it is mandatory to have a response of the system to build the ROM. In the present context of a completely theoretical model, this is a drawback, since one must compute time responses to be in a position of reducing the system. Moreover, it has been underlined by Amabili et al. $(2003,2006)$ that the choice of these time responses is not an easy task that could not be done blindly. By comparison, the asymptotic NNMs method does not need any response of the system, but dynamical properties only, that are here provided by Eq. (9), i.e. after projection of the PDE with the Galerkin method. With the eigenvalues of the linear part (eigenfrequencies $\omega_{j}$ and damping coefficients $\zeta_{j}$ ) in hand and the nonlinear coefficients $g_{i l}^{(j)}$ and $h_{i p k}^{(j)}$, the nonlinear change of coordinates, Eq. (19), can be applied directly to obtain the ROM. As the coefficients in Eq. (19) are computed once and for all, application of the method is easy and not too demanding in terms of computation time. In the next section, all these conclusions will be illustrated with the numerical results.

\section{Numerical results}

The simply supported, water-filled circular cylindrical shell (without imperfections) investigated by Amabili (2003) is considered, with the following dimensions and material properties: $L=520 \mathrm{~mm}, R=149.4 \mathrm{~mm}, h=0.519 \mathrm{~mm}$, $E=2.06 \times 10^{11} \mathrm{~Pa}, \rho=7800 \mathrm{~kg} / \mathrm{m}^{3}, \rho_{F}=1000 \mathrm{~kg} / \mathrm{m}^{3}$ and $v=0.3$. Numerical calculations have been performed for the fundamental mode $(m=1, n=5)$ of the water-filled shell. The natural frequency $\omega_{1,5}$ of this mode is $79.21 \mathrm{~Hz}$, according to Donnell's theory of shells; modal damping $\zeta_{1,5}=0.0017$ is assumed in the Galerkin model for the fundamental mode; for additional modes in the Galerkin model the following modal damping has been assumed: $\zeta_{m n}=\zeta_{15} \omega_{m n} / \omega_{15}$; the same values of modal damping have been used in all the ROMs.

The two reduction methods will be compared in two different cases. First, frequency-response curves, for a moderate value of the amplitude of the forcing, are investigated in the next section. A geometrical interpretation is then proposed, in order to visualize cuts of the subspaces where the dynamics is reduced. Finally, bifurcation diagrams at fixed frequency and for increasing amplitudes of the forcing are studied to check the ability of the methods over a large range of parameter variations with complex dynamical behaviours.

\subsection{Frequency-response curves}

The response of the fundamental mode of the water-filled shell to harmonic point excitation of $3 \mathrm{~N}$ at $\tilde{x}=L / 2$ and $\tilde{\theta}=0$ has been computed by using the conventional Galerkin model with 16 dofs; result is given in Fig. 1 . The solution presents a main branch " 1 " corresponding to zero amplitude of the companion mode $B_{1, n}(t)$; this branch has pitchfork bifurcations (BP) at $\omega / \omega_{1, n}=0.9714$ and at 1.0018 , where branch "2" appears. This new branch corresponds to participation of both $A_{1, n}(t)$ and $B_{1, n}(t)$, giving a travelling wave response. Branch "2" undergoes two Neimark-Sacker (torus) bifurcations (TR), at $\omega / \omega_{1, n}=0.9716$ and 0.9949 . Amplitude-modulated (quasiperiodic) response is indicated in Fig. 1 for $0.9716<\omega / \omega_{1, n}<0.9949$, i.e. bracketed by the two Neimark-Sacker bifurcations.

The quasiperiodic time response of the shell for excitation of $3 \mathrm{~N}$ at frequency $\omega=0.991 \omega_{1, n}$, branch "2", is reported in Fig. 2 for the most significant generalized coordinates. This time response, which is more suitable than simple periodic 

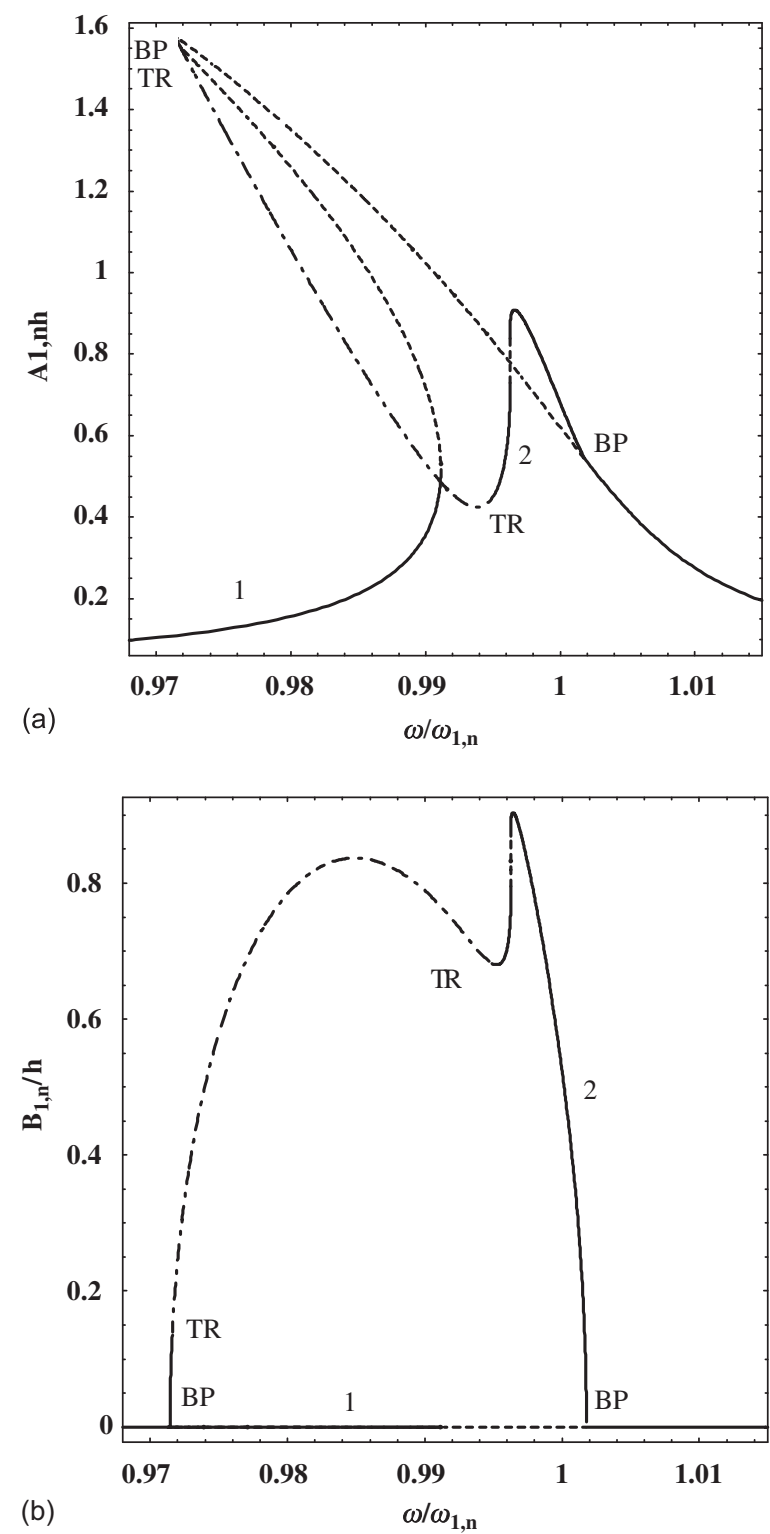

Fig. 1. Maximum amplitude of vibration versus excitation frequency, for excitation of $3 \mathrm{~N}$; conventional Galerkin model, 16 dofs. (a) Maximum amplitude of $A_{1, n}(t)$, driven mode; (b) maximum amplitude of $B_{1, n}(t)$, companion mode; 1, branch " 1 "; 2 , branch "2"; BP, pitchfork bifurcation; TR, Neimark-Sacker (torus) bifurcations. —_, stable periodic solutions; — - —, stable quasi-periodic solutions; - - , unstable periodic solutions.

responses to construct accurate POD models (Amabili et al., 2003), has been used to build a POD model. Both travelling waves around the shell in opposite directions have been taken into account (one obtained by direct integration and the other by changing the sign to the generalized coordinates associated to $\sin (n \theta)$ terms in Eq. (8)) to construct the POD ROM. The optimal number of POMs $\tilde{K}$ to be retained in the ROM can be estimated by plotting $\sum_{i=1}^{\tilde{K}} \lambda_{i} / \sum_{i=1}^{K} \lambda_{i}$ as a function of $\tilde{K}$; three POMs absorb practically all of the shell energy for the response at $\omega / \omega_{1, n}=0.991$; therefore, three POMs are used in the POD model. The coefficients of the main POMs, to be inserted in Eq. (18), are given in Table 1. The first POM is the driven mode, the second is the companion mode and the third is the axisymmetric mode.

Responses obtained by using the conventional Galerkin method (16 dofs) and by using the POD method ( 3 dofs) compare very well for excitation of $3 \mathrm{~N}$, as shown in Fig. 3 for both driven and companion modes; the main difference is 

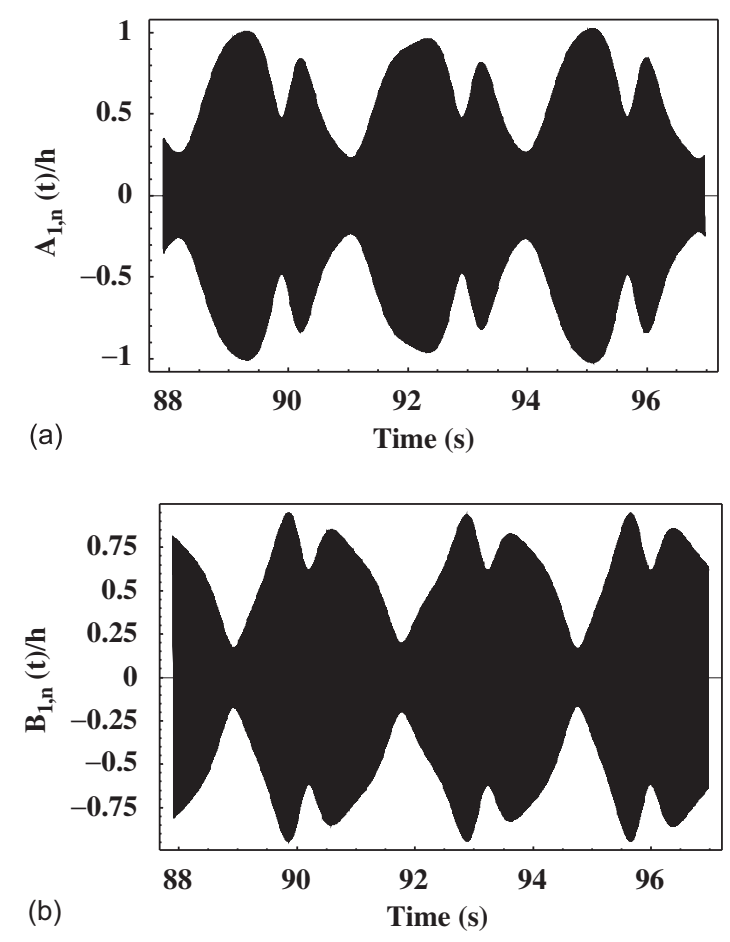

Fig. 2. Time response at excitation frequency $\omega / \omega_{1, n}=0.991$, for excitation of $3 \mathrm{~N}$; conventional Galerkin model, 16 dofs: (a) modal coordinate $A_{1, n}(t)$ associated to the driven mode; (b) modal coordinate $B_{1, n}(t)$ associated to the companion mode.

a slight shift on the right of the first bifurcation point of branch "1". It can also be observed that the natural frequency computed with the POD model is practically identical to the one computed with the Galerkin model. Fig. 3 also shows the response computed with the NNMs method with only two dof. It can be observed that also the response computed with the NNMs method compares very well with the original Galerkin model, with the curves just very slightly shifted in the left and with exact qualitative behaviour. In this case the maximum vibration amplitudes reach about $1.5 h$ for the driven mode and $0.9 h$ for the companion mode.

In order to compare the results also in the time domain, the quasiperiodic responses $\left(\omega / \omega_{1, n}=0.991\right.$, excitation $\left.3 \mathrm{~N}\right)$ computed with the POD and NNMs models are reported in Figs. 4 and 5, respectively; this response is more critical to be reproduced by ROMs than simple periodic responses. Whereas the response computed with the POD is in reasonably good agreement with the one in Fig. 2 obtained with the Galerkin model, the response calculated by using the NNMs model is practically coincident with this.

Fig. 6 has been obtained with the same three models (Galerkin, NNMs and POD for which the same equations obtained with time response for excitation of $3 \mathrm{~N}$ has been used), but for excitation of $8 \mathrm{~N}$. In this case the maximum vibration amplitudes reach about $3 h$ for the driven mode and $2.5 h$ for the companion mode; differences among the three models become much more significant than in the previous case. In particular, the POD model is relatively close to the original Galerkin model, the main difference being the first bifurcation point of branch "1", which is now significantly shifted on the right, giving rise to a significant difference in the qualitative behaviour of the two models. The NNMs model has qualitatively the same behaviour as the original Galerkin model, but the response is significantly shifted on the left, giving rise to the model overestimating the softening nonlinearity of the system.

It can be observed here that the POD model could be improved by using a time response computed for excitation of $8 \mathrm{~N}$ to find the POMs; however, it is interesting here to investigate the robustness of a ROM to changes in the system parameters, and it is therefore convenient to use the same model. On the other hand, the NNMs model is built once and for all, and may not been changed when varying the amplitude of the forcing. The observed differences with the reference solution are the consequences of the two approximations used to build it: asymptotic development and time-invariant manifold. 

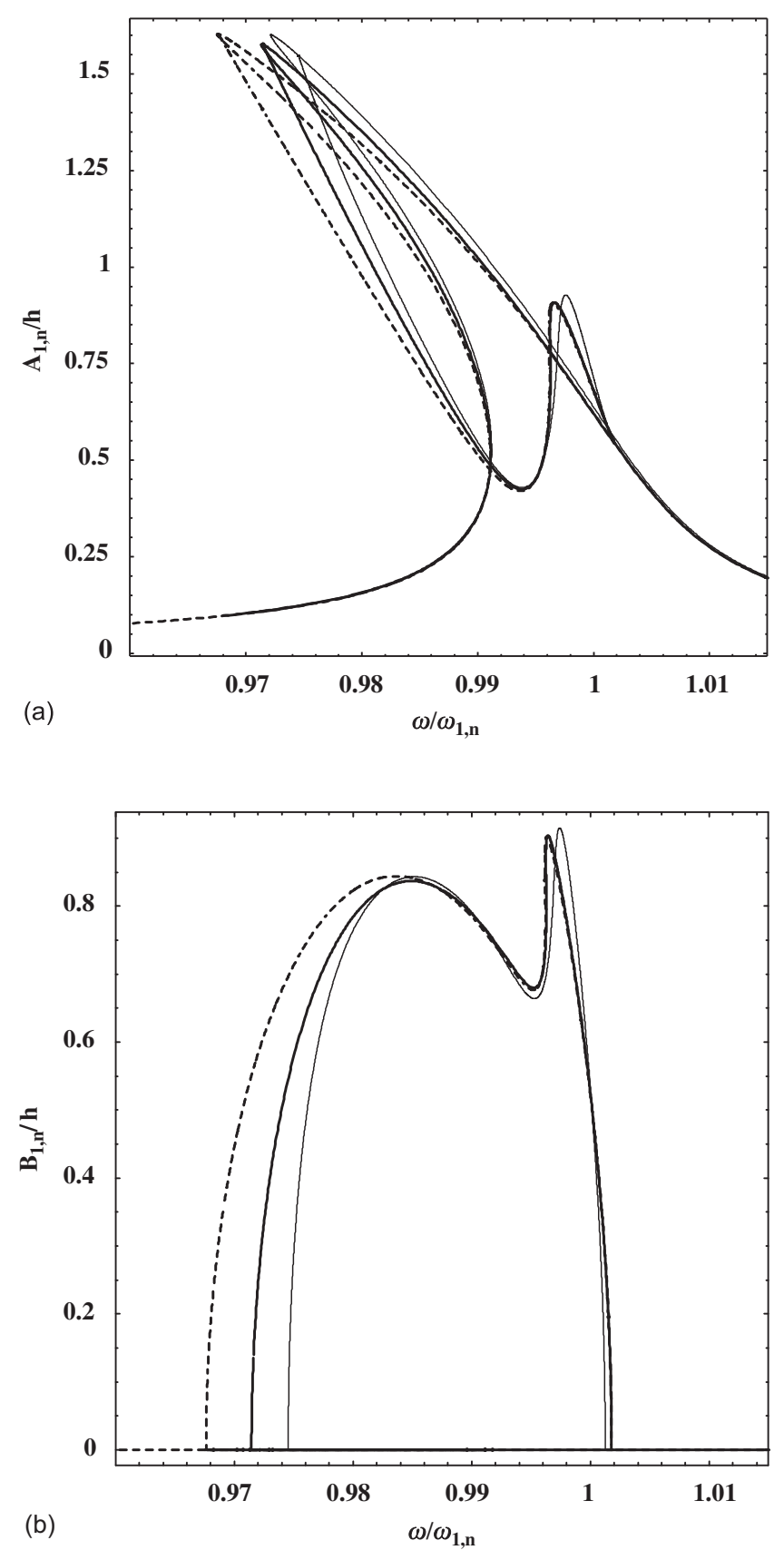

Fig. 3. Maximum amplitude of vibration versus excitation frequency, for excitation of $3 \mathrm{~N}$; conventional Galerkin model, POD model with 3 modes and NNMs model with 2 modes. (a) Maximum amplitude of $A_{1, n}(t)$, driven mode; (b) maximum amplitude of $B_{1, n}(t)$, companion mode. ——, conventional Galerkin model (16 dofs); — , POD model (3 dofs); ——, NNMs model (2 dofs).

It is well known that the contribution of axisymmetric mode, even if it is small compared to the oscillation of the fundamental mode, is fundamental to predict the correct nonlinear behaviour of the shell. It can be interesting to observe that both reduced order models do not need this knowledge a priori, because they act on time responses, in the case of POD, or on the equations of motion, in the case of NNMs, where this information on the system dynamics is included. 

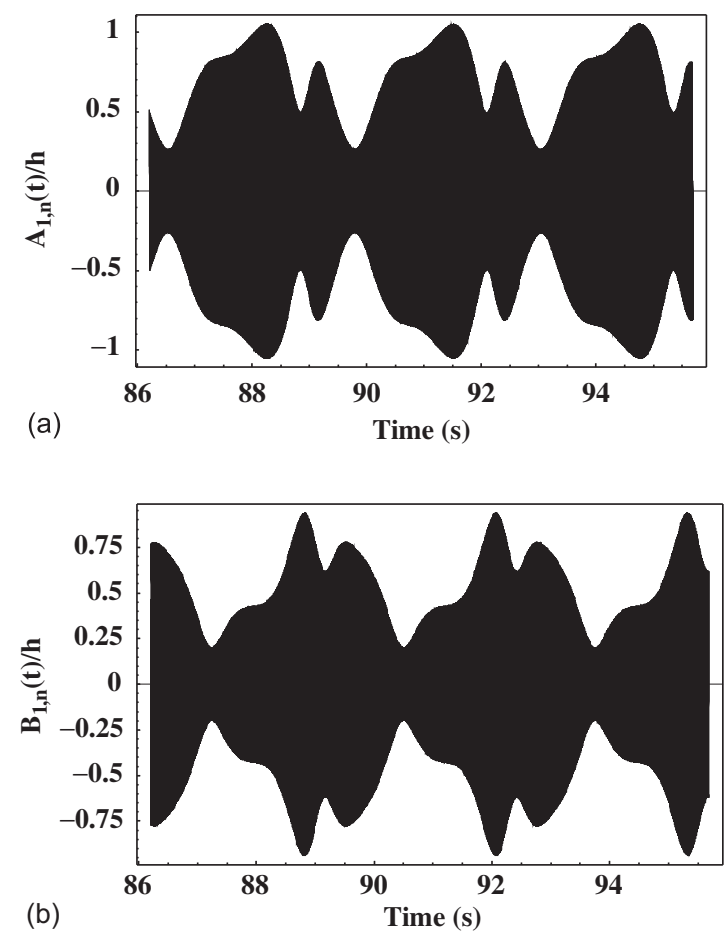

Fig. 4. Time response at excitation frequency $\omega / \omega_{1, n}=0.991$, for excitation of $3 \mathrm{~N}$; POD model, 3 dofs: (a) modal coordinate $A_{1, n}(t)$ associated to the driven mode; (b) modal coordinate $B_{1, n}(t)$ associated to the companion mode.
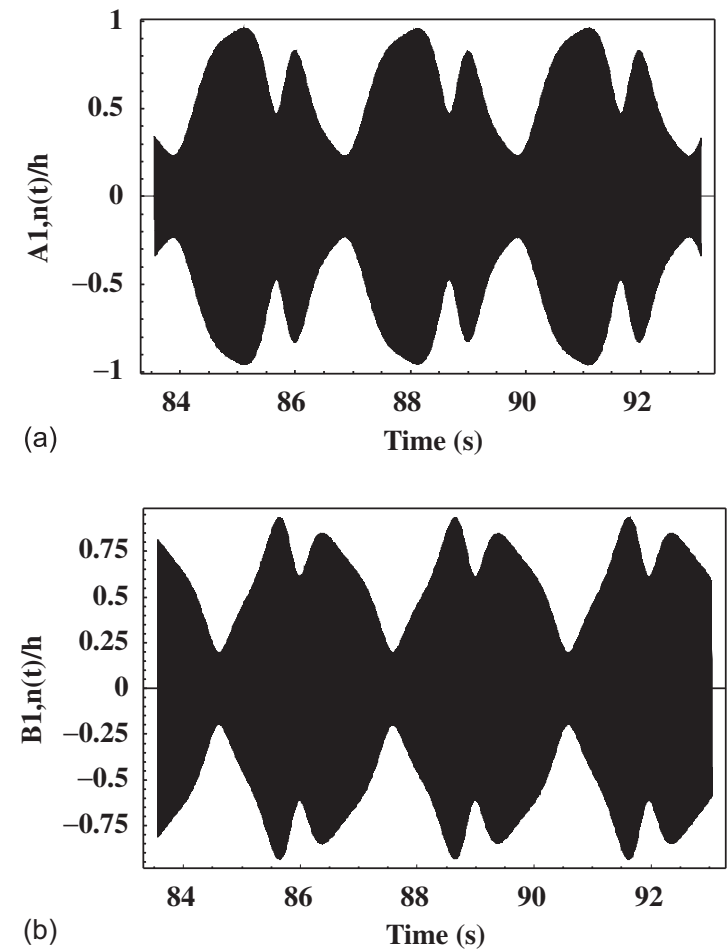

Fig. 5. Time response at excitation frequency $\omega / \omega_{1, n}=0.991$, for excitation of $3 \mathrm{~N}$; NNMs model, 2 dofs: (a) modal coordinate $A_{1, n}(t)$ associated to the driven mode; (b) modal coordinate $B_{1, n}(t)$ associated to the companion mode. 

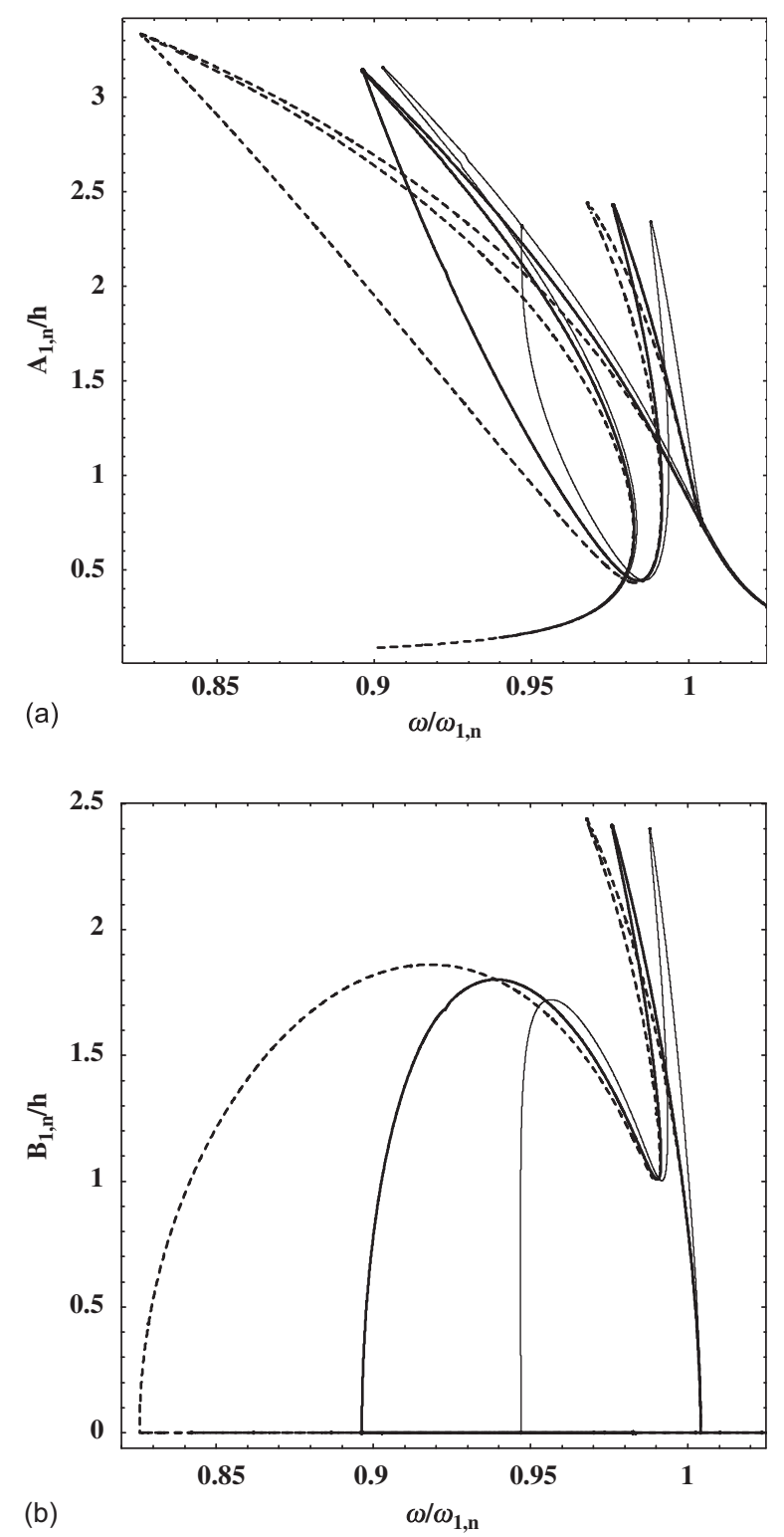

Fig. 6. Maximum amplitude of vibration versus excitation frequency, for excitation of $8 \mathrm{~N}$; conventional Galerkin model, POD model with 3 modes and NNMs model with 2 modes. (a) Maximum amplitude of $A_{1, n}(t)$, driven mode; (b) maximum amplitude of $B_{1, n}(t)$, companion mode. ——, conventional Galerkin model (16 dofs); — , POD model (3 dofs); ——, NNMs model (2 dofs).

\subsection{Geometrical interpretation}

In order to get a geometrical interpretation of the frequency-response curves shown in the previous section, projections and Poincaré sections of the solutions are here proposed. The state space of the reference solution, Eq. (9), is 32-dimensional (16 dofs selected with displacement and velocity as independent variables for each), plus one for the external forcing. Three different time responses are considered for excitation of $3 \mathrm{~N}$ : case (a), $\omega / \omega_{1, n}=0.99$ on branch 1 with no companion mode participation; case (b), $\omega / \omega_{1, n}=0.995$ on branch 2 with companion mode participation and harmonic response; case (c), $\omega / \omega_{1, n}=0.991$ on branch 2 with companion mode participation and quasi-periodic response with amplitude modulations, as shown in Fig. 2. 


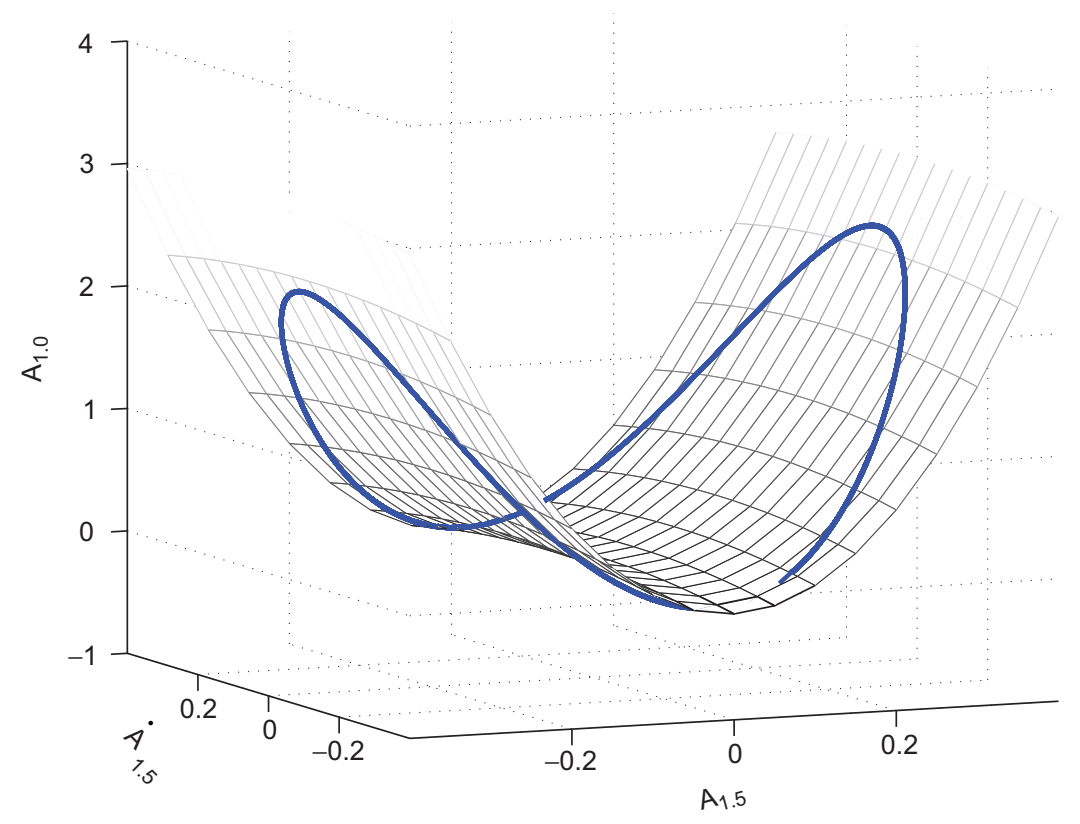

Fig. 7. Projection of the 32-dimensional state space onto the 3-dimensional subspace spanned by $\left(A_{1,5}, \dot{A}_{1,5}, A_{1,0}\right)$. Closed orbit: forced motion for $\omega / \omega_{1, n}=0.99$ and for excitation of $3 \mathrm{~N}$, case (a). Curved surface: invariant manifold corresponding to the first NNM (driven mode).

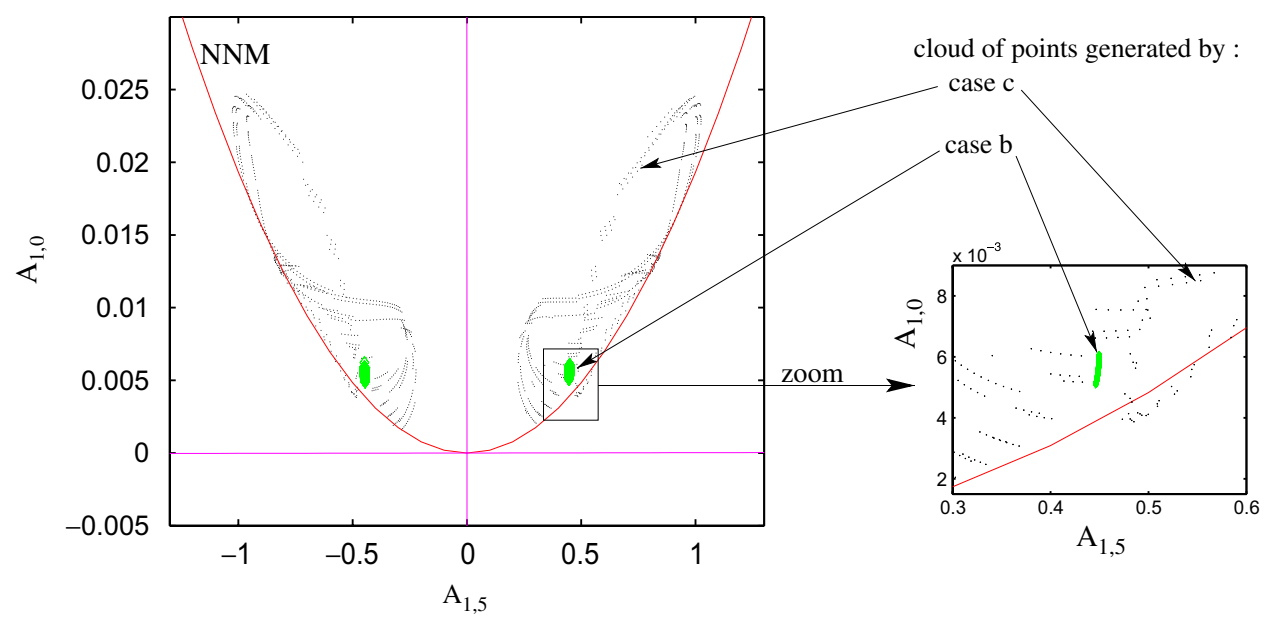

Fig. 8. Poincaré section in $\left(A_{1,5}, A_{1,0}\right)$. Cloud of points: section of the orbits with the Poincaré plane, cases (b) and (c). POD differs very little from the original axis, whereas the cut of the 4-dimensional invariant manifold (curved hyperbolic line) goes right in-between the points (NNMs).

First, a two-dimensional invariant manifold, corresponding to a single NNM, is shown in Fig. 7. This NNM, defined by the coordinates $\left(r_{1}, s_{1}\right)$ introduced in Section 3.3, corresponds to the continuation of the driven asymmetric mode: $\left(A_{1,5}, \dot{A}_{1,5}\right)$. The geometry of the manifold is given by Eq. (19). Keeping $N$ master coordinates (here $N=1$ ) and cancelling the others, Eq. (19) defines a $2 N$-dimensional invariant manifold in state space. The closed orbit is computed by time integration of the reference solution, and corresponds to case (a), where the companion mode is not excited, ensuring a two-dimensional motion. One can observe that the forced motion occurs in the vicinity of the time-invariant manifold, illustrating the quality of the proposed approximation. Once again, the small differences (the orbit goes 
before the manifold for $A_{1,5}>0$, and behind for $A_{1,5}<0$ ) are due to the two approximations used for constructing the subspace.

The dynamics in the vicinity of the first eigenfrequency is essentially governed by the 1:1 internal resonance and the coupling between driven and companion mode. Hence, the dynamics is essentially four-dimensional, so that only Poincaré sections will allow illustration of the geometry of the subspaces used for reduction. Fig. 8 shows such a Poincaré map, where the selected section is the plane $\left(A_{1,5}, A_{1,0}\right)$, chosen in order to show the important contribution of the first axisymmetric mode on the asymmetric motion. Two clouds of points are represented, corresponding to time series computed by the reference model at points $b$ and $c$ of the frequency-response curve. Point $b$ corresponds to $a$ coupled harmonic motion, whereas at point $\mathrm{c}$ the stable solution is quasiperiodic, so that the cloud of points occupies a larger part of the state space. This difference explains in particular why the POD constructed with point $\mathrm{c}$ is better than the one built with point b, as the problem defined by Eq. (14) is numerically better posed when the cloud of points spans a large portion of the state space. Continuous lines in Fig. 8 are the sections of the subspaces provided by POD and NNMs methods, respectively. The new POD axes are given by the $\alpha_{i}$ defined in Eq. (13), whose numerical values are recalled in Table 1. This figure clearly shows why three POD modes are necessary to recover correctly the dynamics. The new POD axes are very close to the original ones, and the cloud of point lie precisely in-between them, with a significant contribution on $A_{1,0}$. Hence, the third POD mode, which corresponds to $A_{1,0}$, must mandatorily be kept in the reduced POD model, otherwise significant dynamical information is discarded.

On the other hand, the section of the four-dimensional invariant manifold (corresponding to two NNMs) is given by the parabolic curve, which goes exactly where the computed points are. This explains why only two NNMs are necessary to recover the dynamics, as the four-dimensional manifold displays an important curvature in the direction of $A_{1,0}$. This feature is general for vibrations of shells. It allows one to use more blindly the Galerkin method in conjunction with NNMs reduction in order to recover correct qualitative behaviour, as this generic coupling between asymmetric motion and axisymmetric contraction is embedded in the NNMs. For example, this property has been used by Touzé and Thomas (2006) in order to derive the type of nonlinearity of shallow spherical shells as a function of its geometry.

Two other Poincaré sections are shown in Fig. 9, showing the contribution of the main forced motion onto $A_{3,0}$ and $A_{1,10}$. Once again, the NNMs provide a better approximation, thanks to the curvature of the reduced subspace. The corresponding POD modes could have been neglected because here the contributions are of the order of $10^{-3}$.

\subsection{Results for large excitations}

The two ROMs are now studied on a large range of variation of the amplitude of the forcing, so as to test them in a very difficult case where numerous different dynamical behaviours have been found to coexist. Poincaré maps have been computed by direct integration of the equations of motion. The excitation frequency $\omega$ has been kept constant, $\omega=0.92 \omega_{1, n}$ (the shell displays softening-type response; therefore, for large excitation, the resonance is obtained for
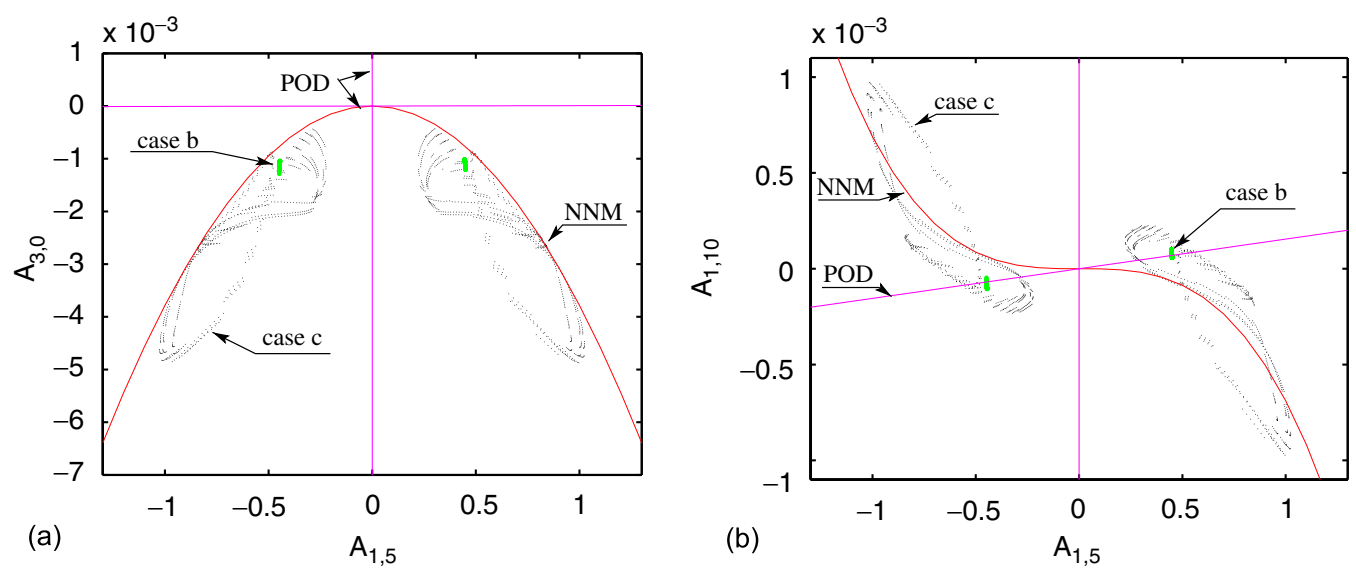

Fig. 9. Poincaré sections; cloud of points: cases (b) and (c). POD axes and NNMs sections in plain lines. (a) Poincaré section in $\left(A_{1,5}, A_{3,0}\right)$; (b) Poincaré section in $\left(A_{1,5}, A_{1,10}\right)$. 

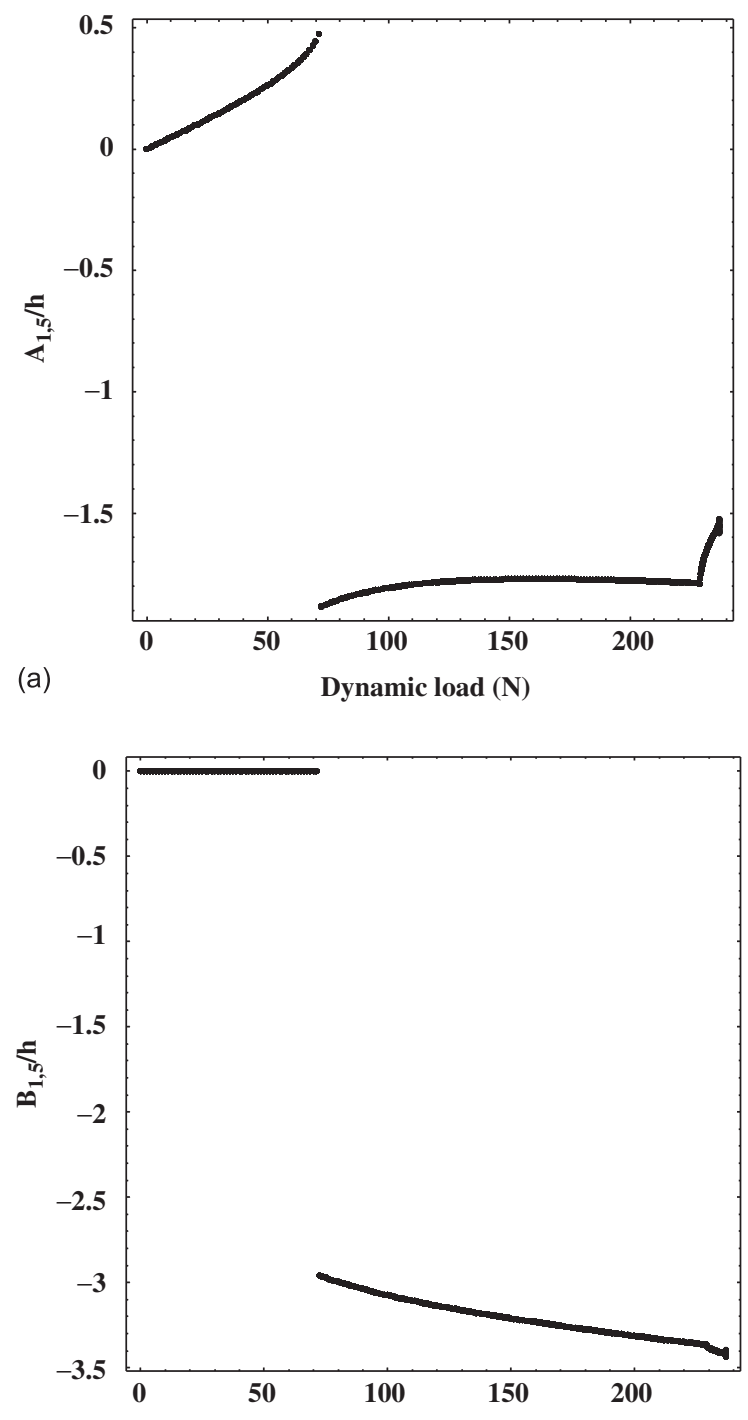

(b)

Dynamic load (N)

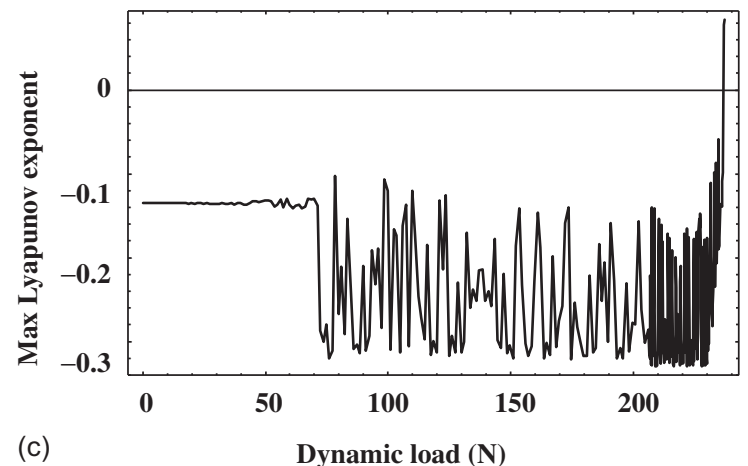

Fig. 10. Bifurcation diagram of Poincaré maps and maximum Lyapunov exponent for the shell under increasing harmonic load with frequency $\omega=0.92 \omega_{1, n}$; NNMs model, 2 dofs. (a) Generalized coordinate $A_{1, n}(t)$, driven mode; (b) generalized coordinate $B_{1, n}(t)$, companion mode; (c) maximum Lyapunov exponent. 
$\left.\omega<\omega_{1, n}\right)$, and the excitation amplitude has been varied between 0 and $600 \mathrm{~N}$. The force range has been divided into 500 steps, so that the force is varied in steps of $1.2 \mathrm{~N}$. Each time the force is changed by a step, 500 periods have been allowed to elapse in order to eliminate the transient motion. The initial condition at the first step is zero displacement and velocity for all the variables. The bifurcation diagrams obtained by all these Poincare maps by using the conventional Galerkin and the several POD models are given by Amabili et al. (2006). The POD model giving the best behaviour among those studied is the one built from chaotic time response at $\omega=0.92 \omega_{1, n}$ and excitation of $550 \mathrm{~N}$; this model has 5 dofs and the coefficients of the main POMs are given in Table 1. Simple periodic motion, a perioddoubling bifurcation, subharmonic response, amplitude modulations and chaotic response have been detected. This indicates the existence of complex nonlinear dynamics for the circular cylindrical shell subject to large harmonic excitation.

In the present study, this POD model (built from chaotic time response) has also been used to evaluate the frequency-response curve for excitation of $3 \mathrm{~N}$. The result is not satisfactory and indicates a wrong, hardening-type nonlinearity. Therefore, a time response obtained for very different system parameters $(550 \mathrm{~N})$ can give an inaccurate POD model.

The bifurcation diagram for $\omega=0.92 \omega_{1, n}$, increasing excitation amplitude up to about $240 \mathrm{~N}$, is given in Fig. 10(a,b) for the NNMs model with two NNMs; the corresponding maximum Lyapunov exponent is shown in Fig. 10(c). The same result has been obtained by using five nonlinear normal modes, as a consequence of the additional three modes having been found numerically to have constant null amplitude in the investigated range. The qualitative and quantitative comparison with the original Galerkin model [see Fig. 4 in Amabili et al. (2006)] is reasonably good, the main difference being that the integration of the equations obtained with the NNMs method is extremely difficult, with small perturbations stopping the integration for divergence of the solution. Moreover, it was impossible to perform the integration over the right-end point shown in Fig. 10, at about $240 \mathrm{~N}$. This difficulty of integration of the NNMs model for large excitations has been related to the different structure of equations (20), where cubic terms involving velocities appear. This ill-natured behaviour of the NNMs ROM is the logical consequence of its definition. Based on a local theory and asymptotic development, the ROM is valid for small values of amplitude of forcing and modal coordinates. On the other hand, the ability of the POD to recover the essential features of the dynamics is due to its global nature. However, the numerical investigation addressed here shows that the local nature of the asymptotic NNMs allows recovering good results for an amplitude of forcing up to $240 \mathrm{~N}$, and for vibration amplitudes up to $3 h$, as shown in Section 4.1. As these values are not at all smaller than one, it can be concluded that the range of validity of the NNM is really greater than what could be expected. Finally, better results could be obtained by bypassing the asymptotic solution, and turning directly to a numerical resolution of the NNMs. However, the main features of the method used, which renders it particularly attractive, would be lost and replaced by an intensive numerical effort.

\section{Conclusions}

Both the proper orthogonal decomposition (POD) and the nonlinear normal modes (NNMs) methods have been verified to be suitable for building ROMs of a water-filled shell. In particular, a larger reduction of the model is possible by using the NNMs method. However, the asymptotic formulation used here in the NNMs method does not make it suitable for studying very large vibration amplitudes, where the POD model performs better.

These results have to be related to the properties of the two methods, discussed in Section 3.4. The nonlinear character of the invariant manifold that defines NNMs allows better reduction than the POD method, which is a linear decomposition. This property has been specifically illustrated in Section 4.2. On the other hand, the global nature of the POD provides very robust results even for complex dynamics, whereas the asymptotic NNMs has been found to fail in recovering these motions. However, it has been found that, despite being an approximation, the qualitative behaviour of the NNMs compares very well with the original solution, until the validity limits are attained. It has been found numerically that these limits are not that small: amplitude of vibration up to $3 h$ and amplitude of the forcing up to $240 \mathrm{~N}$.

Construction of the NNM-based ROM with the asymptotic method is direct and does not need intensive computations, as a single nonlinear change of coordinates, computed once and for all, is required. The method can thus be blindly applied, provided the Galerkin projection has been performed on a large number of modes. On the other hand, for the POD, particular care must be taken in the choice of the time responses used to build it, as already discussed in Amabili et al. (2003).

To conclude, the investigations conducted here show that for moderate vibration amplitude, the asymptotic NNMs method provides more reduced equations that always recover the qualitative behaviour. However, the method must be modified in order to bypass its main limitation, which is due to the asymptotic development. Unfortunately, only 
numerical solutions are possible, thus leading to an intense numerical effort in order to build the ROM. Hence, for very large vibration amplitude and large range of parameter variations, the POD method still performs better due to its global nature.

\section{Acknowledgements}

The first author (M.A.) gratefully acknowledges fundamental cooperation on related topics with Francesco Pellicano, Michael P. Païdoussis and Abhijit Sarkar.

The second author (C.T.) gratefully acknowledges Gérard Iooss and Olivier Thomas for stimulating discussions, respectively, on the mathematical and mechanical aspects of the problems discussed herein.

\section{References}

Amabili, M., 2003. Theory and experiments for large-amplitude vibrations of empty and fluid-filled circular cylindrical shells with imperfections. Journal of Sound and Vibration 262, 921-975.

Amabili, M., Païdoussis, M.P., 2003. Review of studies on geometrically nonlinear vibrations and dynamics of circular cylindrical shells and panels, with and without fluid-structure interaction. Applied Mechanics Reviews 56 (4), 349-381.

Amabili, M., Sarkar, A., Païdoussis, M.P., 2003. Reduced-order models for nonlinear vibrations of cylindrical shells via the proper orthogonal decomposition method. Journal of Fluids and Structures 18, 227-250.

Amabili, M., Sarkar, A., Païdoussis, M.P., 2006. Chaotic vibrations of circular cylindrical shells: Galerkin versus reduced-order models via the proper orthogonal decomposition method. Journal of Sound and Vibration 290, 736-762.

Aubry, N., Holmes, P., Lumley, J.L., Stone, E., 1988. The dynamics of coherent structures in the wall region of a turbulent boundary layer. Journal of Fluid Mechanics 192, 115-173.

Azeez, M.F., Vakakis, A.F., 2001. Proper orthogonal decomposition (POD) of a class of vibroimpact oscillations. Journal of Sound and Vibration 240, 859-889.

Bellizzi, S., Bouc, R., 2005. A new formulation for the existence and calculation of nonlinear normal modes. Journal of Sound and Vibration 287, 545-569.

Breuer, K.S., Sirovich, L., 1991. The use of the Karhunen-Loève procedure for the calculation of linear eigenfunctions. Journal of Computational Physics 96, 277-296.

Carr, J., 1981. Applications of Centre Manifold Theory. Springer, New York.

Doedel, E.J., Champneys, A.R., Fairgrieve, T.F., Kuznetsov, Y.A., Sandstede, B., Wang, X., 1998. AUTO 97: Continuation and Bifurcation Software for Ordinary Differential Equations (with HomCont). Concordia University, Montreal, Canada.

Elphick, C., Tirapegui, E., Brachet, M., Coullet, P., Iooss, G., 1987. A simple global characterization for normal forms of singular vector fields. Physica D 29, 95-127.

Evensen, D.A., 1967. Nonlinear flexural vibrations of thin-walled circular cylinders. NASA TN D-4090, Washington DC, USA.

Georgiou, I.T., 2005. Advanced proper orthogonal decomposition tools: using reduced order models to identify normal modes of vibration of slow invariant manifolds in the dynamics of planar nonlinear rods. Nonlinear Dynamics 41, 69-110.

Georgiou, I.T., Schwartz, I., Emaci, E., Vakakis, A., 1999. Interaction between slow and fast oscillations in an infinite degree-offreedom linear system coupled to nonlinear subsystem: theory and experiment. Journal of Applied Mechanics 66, 448-459.

Guckenheimer, J., Holmes, P., 1983. Non-linear Oscillations, Dynamical Systems and Bifurcations of Vector Fields. Springer, New York.

Iooss, G., Adelmeyer, M., 1998. Topics in Bifurcation Theory, second ed. World Scientific, New York, USA.

Jézéquel, L., Lamarque, C.H., 1991. Analysis of non-linear dynamical systems by the normal form theory. Journal of Sound and Vibration $149,429-459$

Jiang, D., Pierre, C., Shaw, S., 2005a. The construction of non-linear normal modes for systems with internal resonance. International Journal of Non-Linear Mechanics 40, 729-746.

Jiang, D., Pierre, C., Shaw, S., 2005b. Nonlinear normal modes for vibratory systems under harmonic excitation. Journal of Sound and Vibration 288, 791-812.

Kerschen, G., Feeny, B.F., Golinval, J.-C., 2003. On the exploitation of chaos to build reduced-order models. Computer Methods in Applied Mechanics and Engineering 192, 1785-1795.

Kerschen, G., Golinval, J.-C., Vakakis, A.F., Bergman, L.A., 2005. The method of proper orthogonal decomposition for dynamical characterization and order reduction of mechanical systems: an overview. Nonlinear Dynamics 41, 147-169.

King, M.E., Vakakis, A.F., 1994. Energy-based formulation for computing nonlinear normal modes in undamped continuous systems. Journal of Vibration and Acoustics 116, 332-340.

Lacarbonara, W., Rega, G., Nayfeh, A.H., 2003. Resonant non-linear normal modes. Part I: analytical treatment for structural onedimensional systems. International Journal of Non-Linear Mechanics 38, 851-872.

Mikhlin, Yu.V., 1995. Matching of local expansions in the theory of non-linear vibrations. Journal of Sound and Vibration 182, $577-588$

Pellicano, F., Amabili, M., Païdoussis, M.P., 2002. Effect of the geometry on the non-linear vibration of circular cylindrical shells. International Journal of Non-Linear Mechanics 37, 1181-1198. 
Pesheck, E., Pierre, C., Shaw, S., 2002. A new Galerkin-based approach for accurate non-linear normal modes through invariant manifolds. Journal of Sound and Vibration 249, 971-993.

Poincaré, H., 1892. Les méthodes nouvelles de la mécanique céleste. Gauthiers-Villars, Paris.

Rosenberg, R.M., 1966. On non-linear vibrations of systems with many degrees of freedom. Advances in Applied Mechanics 9, $155-242$.

Sarkar, A., Païdoussis, M.P., 2003. A compact limit-cycle oscillation model of a cantilever conveying fluid. Journal of Fluids and Structures 17, 525-539.

Sarkar, A., Païdoussis, M.P., 2004. A cantilever conveying fluid: coherent modes versus beam modes. International Journal of NonLinear Mechanics 39, 467-481.

Shaw, S., Pierre, C., 1991. Non-linear normal modes and invariant manifolds. Journal of Sound and Vibration 150, $170-173$.

Shaw, S.W., Pierre, C., 1993. Normal modes for non-linear vibratory systems. Journal of Sound and Vibration 164, 85-124.

Sirovich, L., 1987. Turbulence and dynamics of coherent structures, Part I: coherent structures. Quarterly Journal of Applied Mathematics 45, 561-571.

Slater, J.C., 1996. A numerical method for determining nonlinear normal modes. Nonlinear Dynamics 10, 19-30.

Touzé, C., Amabili, M., 2006. Non-linear normal modes for damped geometrically non-linear systems: application to reduced-order modeling of harmonically forced structures. Journal of Sound and Vibration 298, 958-981.

Touzé, C., Thomas, O., 2006. Non-linear behaviour of free-edge shallow spherical shells: effect of the geometry. International Journal of Non-Linear Mechanics 41, 678-692.

Touzé, C., Thomas, O., Chaigne, A., 2004. Hardening/softening behaviour in nonlinear oscillations of structural systems using nonlinear normal modes. Journal of Sound and Vibration 273, 77-101.

Vakakis, A.F., Manevich, L.I., Mikhlin, Yu.V., Pilipchuk, V.N., Zevin, A.A., 1996. Normal Modes and Localization in Non-Linear Systems. Wiley, New York.

Wolfram, S., 1999. The Mathematica Book, fourth ed. Cambridge University Press, Cambridge, UK.

Zahorian, S.A., Rothenberg, M., 1981. Principal component analysis for low-redundancy encoding of speech spectra. Journal of the Acoustical Society of America 69, 519-524. 